\title{
Post Triangular Rewiring Method for Shorter RRT Robot Path Planning
}

Jin-Gu Kang and Jin-Woo Jung

Department of Computer Science and Engineering, Dongguk University, Seoul, Korea

\section{]jfis}

Received: Jul. 2, 2021

Revised : Jul. 2, 2021

Accepted: Sep. 9, 2021

Correspondence to: Jin-Woo Jung (jwjung@dongguk.edu)

(T)The Korean Institute of Intelligent Systems

(c) This is an Open Access article distributed under the terms of the Creative Commons Attribution Non-Commercial License http://creativecommons.org/licenses/by-nc / 3.0/) which permits unrestricted noncommercial use, distribution, and reproduction in any medium, provided the original work is properly cited.

\begin{abstract}
This paper proposed the "post triangular rewiring" method that minimizes the sacrifice of planning time and overcomes the limit of optimality of sampling-based algorithms such as the rapidly exploring random tree (RRT) algorithm. The proposed "post triangular rewiring" method creates a path closer to the optimal path than the RRT algorithm before application, using the triangular inequality principle. Experiments were conducted to verify the performance of the proposed method. When the proposed method is applied to the RRT algorithm, the optimality efficiency increases compared to the planning time.
\end{abstract}

Keywords: Robot path planning, RRT, Rewiring, Triangular inequality

\section{Introduction}

Path planning [1-3] creates or plans a path for a mobile robot to efficiently move from a starting point to a destination point in Euclidean space, avoiding obstacles, with optimality, clearance, and completeness. Optimality implies always ensuring the planning of a path with the optimal path length. Clearance indicates how low the probability of collision between obstacles and the mobile robot is. Completeness indicates whether a path can always be planned in the presence of a solution (that is, whether a path can be created from the starting point to the destination point without colliding with obstacles).

This paper proposed a treatment method for a sampling-based rapidly exploring random tree (RRT) algorithm [4] that does not guarantee optimality. The RRT algorithm can be summarized as a method of planning a path by repeating the act of inserting a randomly sampled position as a child node in a tree with the starting point as the root node until the destination point is reached. With this algorithm, the tree extends in the shape of a stochastic fractal and has a process of determining the destination point.

The sampling-based algorithms [5--10], including the RRT algorithm, have the advantage of being able to plan a path in less time with less computation than the classical path planning algorithms, such as visibility graph-based [11], cell decomposition-based [12], and potential field-based [13] algorithms. However, they do not guarantee optimality, and have the disadvantage that completeness is guaranteed probabilistically. The latter is also called probabilistic completeness [14], which implies that completeness is guaranteed when the number of random samples is infinite, but may not be guaranteed when the number of random samples is finite. The purpose of this study is to analyze the path planning of the RRT algorithm, which guarantees completeness and shows performance closer to optimality than related works.

The proposed "post triangular rewiring" method is effective in path planning algorithms 
that do not guarantee optimality, such as the RRT algorithm, and show locally piecewise linear shape and can be applied as a post-processing method after a path is planned using such an algorithm.

Indeed, because the proposed method has the greatest advantage of the sampling-based path planning algorithm, that is, the fast planning speed owing to the small amount of computation compared to the classical path planning algorithms [5], the amount of computation added by the proposed method should not be large compared to that of the RRT algorithm.

The performance of the proposed method was verified through mathematical modeling. The planning time and path length of the first complete path (the path that first reaches a destination point from a starting point) were compared and analyzed through simulation when the method proposed in various environments was applied to the RRT algorithm and related works and when the method was not applied.

\section{Rapidly Exploring Random Tree}

The RRT algorithm is a representative algorithm of the samplingbased path planning algorithm proposed by LaValle in 1998 [4]. It is useful for planning a path considering the conditions of non-holonomic constraints and is designed to have high degrees of freedom.

When a random sample is generated in the configuration space, as shown in Figure 1, the nearest node to the position of the random sample is determined among the nodes constituting the tree with the starting point as the root node. A new node is created at a position away from the node by a step length in the direction of the random sample position and inserted into the tree. If the random sample position is closer than the step length, a new node is created at the random sample position and inserted into the tree. This tree extension process is repeated until the destination point is reached.

\section{Proposed Post Triangular Rewiring Method}

The proposed "post triangular rewiring" method can be applied to path planning algorithms that do not guarantee optimality, such as RRT, and rewired based on the triangular inequality principle [6].

It is a post-processing method that is applied after a path is planned using such an algorithm. As shown in Figure 2(a), when there is no obstacle between the current focusing node and its grandparent node (collision-free), a shorter path can be

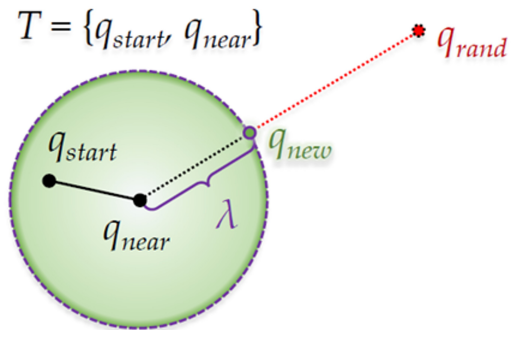

Figure 1. Process of the RRT algorithm: In the case of creating new node $q_{\text {new }}$ at a position separated by step length $\lambda$ in the direction of the random sample position $\left(q_{\text {rand }}\right)$ based on the $q_{\text {near }}$ node (position) nearest to the random sample position $\left(q_{\text {rand }}\right)$ in tree $T$ with starting point $q_{\text {start }}$ as the root node.

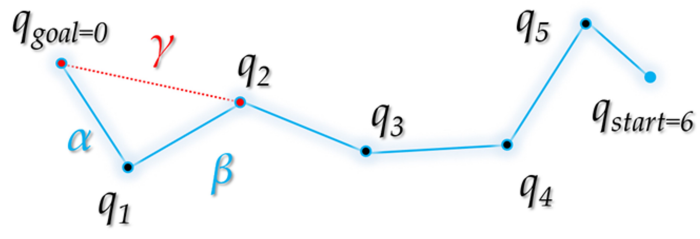

(a)

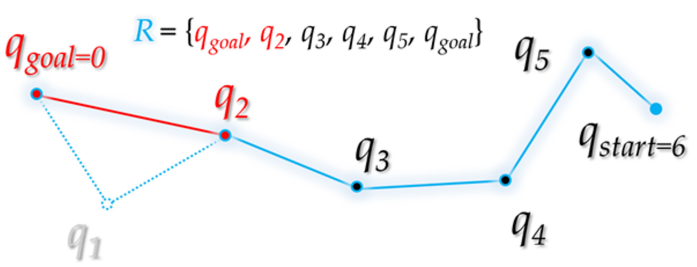

(b)

Figure 2. Summary of the post triangular rewiring method: (a) When line segment $\gamma$ with node $q_{0}$ and its grandparent node $q_{2}$ in tree $R$ is free from obstacle collision (Distance: $\gamma<\alpha+\beta$ ), (b) Grandparent node $q_{2}$ of node $q_{0}$ is connected as the parent node of node $q_{0}$, and parent node $q_{1}$ is deleted from the tree.

created based on the triangular inequality, as shown in Figure 2(b). The focusing node and the grandparent node of the node are connected as a parent node and rewired, such as deleting the parent node. Therefore, when the proposed post triangular rewiring method is applied, it is possible to modify the path closer to the optimum than the original path of the RRT algorithm.

Figures 3(a) and 3(b) illustrate an example of rewiring [6], which can be expressed as Eq. (1).

$$
q_{\text {ancestor }}=\xi\left(q_{\text {parent }}\right)=\xi^{2}\left(q_{\text {child }}\right) .
$$

Here, $\xi()$ represents a function that receives the node as a variable and returns the parent node of that node. The $n$-squared $(n \geq 0)$ of the $\xi()$ function can be expressed as $\xi^{n}\left(q_{i}\right)=$ 


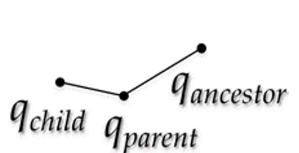

(a)

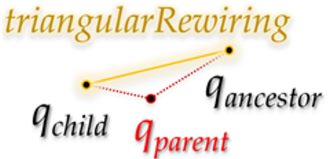

(b)

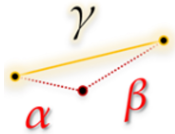

(c)
Figure 3. (a) Part of a tree consisting of node $q_{\text {child }}$, its parent node $q_{\text {parent }}$, and node $q_{\text {ancestor }}$, the parent node of $q_{\text {parent }}$. (b) If there is no obstacle between $q_{\text {child }}$ and $q_{\text {ancestor }}$, delete the edge connecting to $q_{\text {parent }}$ in each node. (c) Edge $\alpha$ connecting $q_{\text {child }}$ and $q_{\text {parent }}$, edge $\beta$ connecting $q_{\text {parent }}$ and $q_{\text {ancestor }}$, and edge $\gamma$ connecting $q_{\text {child }}$ and $q_{\text {ancestor }}$.

$(\overbrace{\xi \circ \xi \circ \cdots \circ \xi})^{n}\left(q_{i}\right)$, and if $n$ is $0, \xi^{0}\left(q_{i}\right)=q_{i}$ holds. That is, $m_{F}\left(q_{i}, k\right)(k>0)$ becomes the midpoint between $m_{F}\left(q_{i}, k-\right.$ $1)$ and $\xi\left(q_{i}\right)$. At this time, $d$ is $\left(d_{k}-1\right) / 2$.

In addition, by substituting into the triangular inequality in (1), it can be expressed as shown in Figure 3(c) and Equation (2).

$$
\alpha+\beta \geq \gamma
$$

Equation (3) provides the distance relation between ancestor nodes based on $q_{\text {child }}$.

$$
\begin{aligned}
& D\left(q_{\text {child }}, \xi\left(q_{\text {child }}\right)\right)+D\left(\xi\left(q_{\text {child }}\right), \xi^{2}\left(q_{\text {child }}\right)\right) \\
& \geq D\left(q_{\text {child }}, \xi^{2}\left(q_{\text {child }}\right)\right) .
\end{aligned}
$$

Equation (3) is the distance $D()$ expressed in (1) between the $n$-th ancestor nodes based on $q_{\text {child }}$ in (2), which represents the distance between each node as a triangular inequality, and then substituted. Here, $D()$ represents the distance between two points, that is, Equations (1)-(3) locally show that the path becomes shorter when rewired.

Algorithms 1 and 2 present the pseudocode of the proposed post triangular rewiring method.

The input value of the post triangular rewiring method includes path $R$ planned using the path planning algorithm, such as the RRT algorithm, obstacle area information $C$, and threshold value $\varepsilon$ of the minimum clearance.

$f_{\text {modify }}$ is a variable that determines whether input path $R$ has been modified by this method, and if the path is modified even once, the entire process is repeated. If path modification does not occur in the repeated process, the algorithm is terminated. $t$ refers to the index of the waypoint of $R$, which is currently the focus. If $t$ is 0 , it indicates the starting point, which is the first point of $R$.
Algorithm 1. Pseudocode of the proposed "post triangular rewiring" method

\section{Input:}

$R \leftarrow$ path from $\{R R T / \ldots\}$

$C \leftarrow$ position set of all (measured) boundary points in all (known) obstacles

$\varepsilon \leftarrow$ threshold value of minimum Clearance

\section{Output:}

$R \leftarrow$ modified path $R$

\section{Initialize:}

$f_{\text {modify }} \leftarrow$ true

Procedure postTriangular Rewire

Begin

1 While $f_{\text {modify }}$ Do

$2 f_{\text {modify }} \leftarrow$ false $/ /$ is the path modified

$3 t \leftarrow 0 / /$ index of the currently focused point

$4 \quad q_{\text {child }} \leftarrow$ first point in $R$

$5 \quad q_{\text {parent }} \leftarrow$ next point of $q_{\text {child }}$ in $R$

6 While not $\left[q_{\text {parent }}\right.$ is last point in $\left.R\right]$ Do

$7 \quad q_{\text {ancestor }} \leftarrow$ next point of $q_{\text {parent }}$ in $R$

8 If not $i s$ Trapped $\left(q_{\text {child }}, q_{\text {ancestor }}, C\right)$ Then

$9 \quad R \leftarrow \operatorname{rewire}\left(R, \varepsilon, t, f_{\text {modify }}\right)$

10

11

Else

$t \leftarrow t+1$

$q_{\text {child }} \leftarrow t$-th point in $R$

$q_{\text {parent }} \leftarrow$ next point of $q_{\text {child }}$ in $R$

End

$\overline{\text { Algorithm 2. Pseudocode of the "rewire" function from the }}$ proposed method

\section{Input:}

$R \leftarrow$ path $R$ from postTriangular Rewire

$t \leftarrow$ point index $t$ from postTriangular Rewire

$f_{\text {modify }} \leftarrow$ boolean $f_{\text {modify }}$ from postTriangular Rewire

\section{Output:}

$R \leftarrow$ modified path $R$

$f_{\text {modify }} \leftarrow$ result of boolean $f_{\text {modify }} / /$ return by reference

Procedure rewire From postTriangular Rewire

Begin

$1 \quad q_{\text {child }} \leftarrow t$-th point in $R$

$2 \quad q_{\text {parent }} \leftarrow$ next point of $q_{\text {child }}$ in $R$

$3 q_{\text {ancestor }} \leftarrow$ next point of $q_{\text {parent }}$ in $R$

$4 R \leftarrow$ Delete path $<q_{\text {child }}, q_{\text {parent }}>$ and path $<q_{\text {parent }}$, $q_{\text {ancestor }}>$ from $R$

$5 \quad R \leftarrow$ Insert path $<q_{\text {child }}, q_{\text {ancestor }}>$ to $R$

$6 f_{\text {modify }} \leftarrow$ true

End

In $R$, when the first focusing point is $q_{c h i l d}$, its next point is $q_{\text {parent }}$, whose next point is $q_{\text {ancestor }}$, it is determined whether the distance between $q_{\text {child }}$ and $q_{\text {ancestor }}$ is free from obstacle 


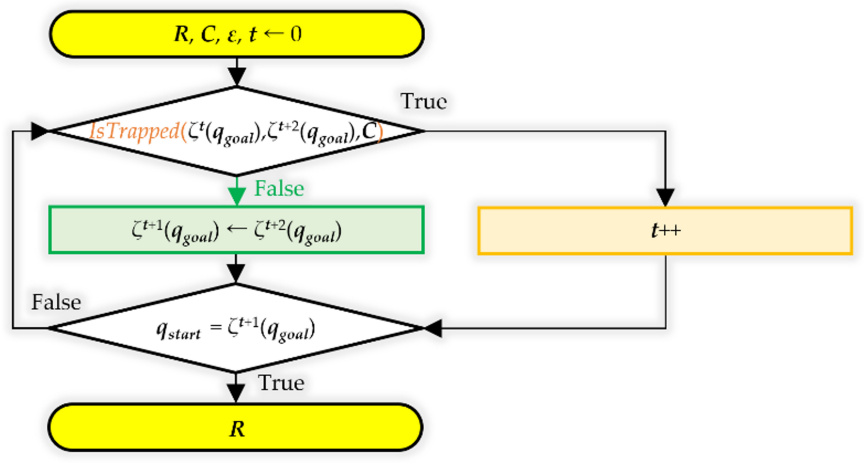

Figure 4. Flow chart of the proposed post triangular rewiring method.

collision (isTrapped() function). If it is free from collisions, it calls rewire(). rewire() connects $q_{\text {child }}$ and $q_{\text {ancestor }}$, and the $q_{\text {parent }}$ between them is deleted from the path. If $R$ and $t$ are updated owing to rewire (), update $q_{\text {child }}$ (the $t$-th waypoint of $R), q_{\text {parent }}$, and $q_{\text {ancestor }}$ accordingly. If $q_{\text {parent }}$ is the last point in $R$, then check $f_{\text {modify }}$. Otherwise, repeat the above process again for the updated $q_{\text {child }}$ and $q_{\text {ancestor }}$.

Here, path modification by rewire() deletes the waypoints and creates a path closer to the optimal path. The input value of rewire() of the post triangular rewiring method includes path $R$, focusing point index $t$, and path modification $f_{\text {modify }}$. Rewiring is performed on the $t$-th waypoint $q_{c h i l d}$ of $R$, next point $q_{\text {parent }}$, and next point $q_{\text {ancestor }}$ of that point. First, the path between $q_{\text {child }}$ and $q_{\text {parent }}$ and the path between $q_{\text {parent }}$ and $q_{\text {ancestor }}$ are deleted. Then, the path between $q_{\text {child }}$ and $q_{\text {ancestor }}$ is inserted. Finally, $f_{\text {modify }}$ returns "true" because the path has been modified.

Figure 4 presents the overall flow chart of the proposed post triangular rewiring method. Here, $\xi^{t}\left(q_{\text {goal }}\right)$ denotes the $t$-th next waypoint from the starting point $q_{\text {goal }}$ of path $R$, and $\xi^{t+n}\left(q_{\text {goal }}\right)$ denotes the $n$-th next waypoint in $\xi^{t}\left(q_{\text {goal }}\right)$. That is, there are $n$ waypoints between $\xi^{t}\left(q_{\text {goal }}\right)$ and $\xi^{t+n}\left(q_{\text {goal }}\right)$.

\section{Experimental Results}

To verify the performance of the proposed post triangular rewiring method, the path between an RRT in various environments through simulation and the RRT algorithm to which the proposed method is applied, path planning results were compared.

The compared performance measures were the average of all trials when each algorithm was repeated 100 times (the sampling position was changed for each trial), path length (pixel), and planning time (ms) of the first complete path (from the start- ing point to the destination point until the first path is planned).

Figure 8 depicts the four environmental maps used in the experiment. Here, the green circle (S) indicates the starting point, and the purple circle $(\mathrm{G})$ indicates the destination point. A black polygon with a yellow border (blue in the experimental results) represents an obstacle. The size of all environmental maps was $600 \times 600$ pixels, and the step length was 30 pixels.

In the related works of the path planning algorithm, various environmental maps were considered and utilized to confirm the performance of the proposed method. It is important to determine the environmental map to use because the efficiency of the performance measures expected during the experiment is slightly different depending on the composition, such as the number, arrangement, or shape of obstacles. In this study, the four environmental maps shown in Figures 5-8 were used to verify the performance of the proposed method. These maps are a part of the experimental environment proposed by Han and Seo [15] in 2017, and the efficiency of the following characteristics and performance measures is expected for each map.

Map 1 of Figure 5 appears to be an environment that is efficient to verify optimality and completeness, and it is an environment unfavorable to sampling-based path planning algorithms such as the RRT algorithm. Because the probability of determining a solution is low, several samplings are required. Map 2 of Figure 6 is an environment that is efficient to verify the optimality and completeness of the path planning algorithm. Map 3 of Figure 7 is an environment that is efficient to verify the optimality of the path planning algorithm and the planning time because it comprises obstacles (50 squares) that are close to curved. The expensive classical path planning algorithm is unfavorable. Map 4 of Figure 8 is an environment that is efficient to verify the completeness and planning time of the path planning algorithm, and is an environment unfavorable to sampling-based path planning algorithms such as the RRT algorithm. Because the sampling-based path planning algorithm depends on probabilistic completeness, the number of samples and planning time required becomes extremely large and long, respectively, as there are narrow or few entrances in the direction to the destination point.

Table 1 lists the performance of the computer used in the simulation. The simulator used for the simulation [6] was developed based on C\# WPF (Microsoft Visual Studio Community 2019 Version 16.1.6 Microsoft .NET Framework Version 4.8.03752), and only a single thread was used for the calculations, except for the visual part. Indeed, there might be differences in the planning time during simulation depending on the 


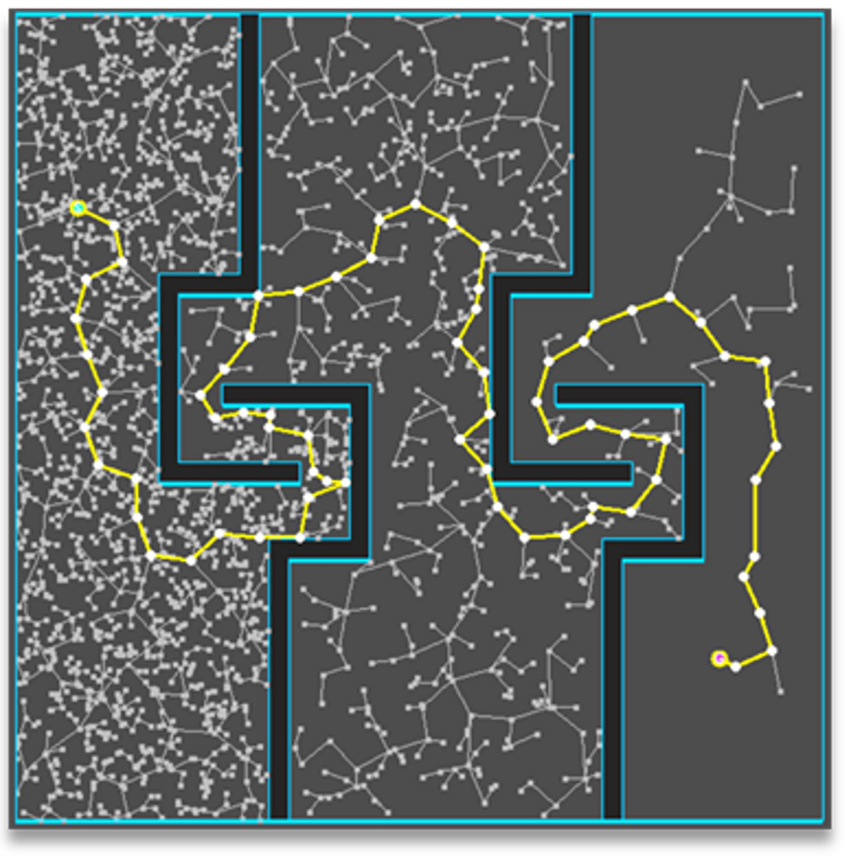

(a)

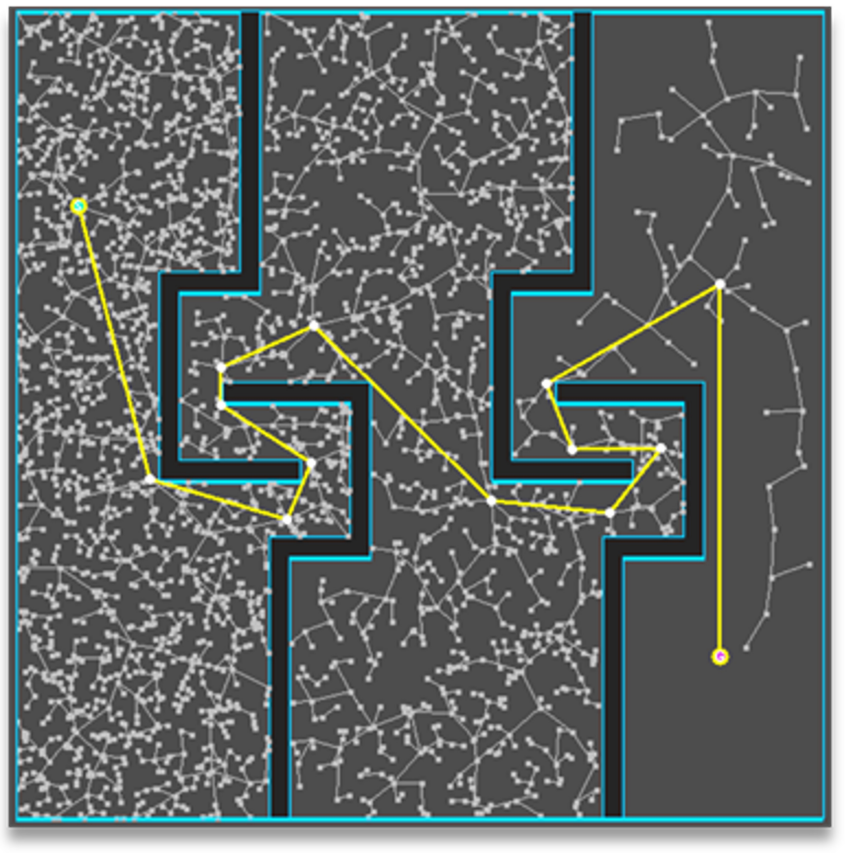

(b)

Figure 5. Experimental results of Map 1: (a) RRT and (b) proposed.

computer performance. Therefore, in this study, the planning time was not compared absolutely, but relatively, based on the RRT algorithm.

The experimental results (path length and planning time) were analyzed when the "post triangular rewiring" method was
Table 1. Computer performance for simulation

\begin{tabular}{cc}
\hline $\mathbf{H} / \mathbf{W}$ & Specification \\
\hline $\mathrm{CPU}$ & Intel Core i7-6700K 4.00 GHz (8 CPUs) \\
RAM & $32768 \mathrm{MB}$ (32 GB DDR4) \\
\hline
\end{tabular}

Table 2. Experimental results of Map 1

\begin{tabular}{lcc}
\hline \multicolumn{1}{c}{ Performance } & RRT & Proposal \\
\hline Path length (pixel) & $1932(100 \%)$ & $1403(72 \%)$ \\
Planning time (ms) & $687(100 \%)$ & $688(100 \%)$ \\
\hline
\end{tabular}

The parentheses on the right of each number (average of repeated 100 times) are relative ratios based on $100 \%$ RRT (values less than 1 are counted as 1).

applied to the RRT algorithm, and its path planning results in the four environmental maps were presented in the experimental environment.

In each map, a figure of the path planning (in the case of a single trial) result for each algorithm is shown, and the results of the experiment (when it is repeated) on the performance measures are presented as numerical values in each table (the figure for each algorithm is not the result of repeated trials). As for the repeated trials, there might be a large difference between the performance observed visually and the numerical results shown in the table. The figure illustrated the shape of the planned path for each algorithm. In addition, it was determined whether there was a section in which the piecewise linear shape path was smoothed by the proposed "post triangular rewiring" method.

Figure 5 shows the path planning results for Map 1 among the presented environmental maps for each algorithm. Visually, the path of the "post triangular rewiring" method appears to be the shortest.

Table 2 presents the path planning results for Map 1 (average when repeated 100 times) among the presented environmental maps for each algorithm. The path length after applying the post triangular rewiring method was $72 \%$ (1403/1932) compared to that of the RRT, which was the shortest, and the planning time was similar.

Figure 6 shows the path planning results for Map 2 among the presented environmental maps for each algorithm. Visually, the path of the "post triangular rewiring" method appears to be the shortest.

Table 3 presents the path planning results for Map 2 (average when repeated 100 times) among the presented environmental maps for each algorithm. The path length after applying the 


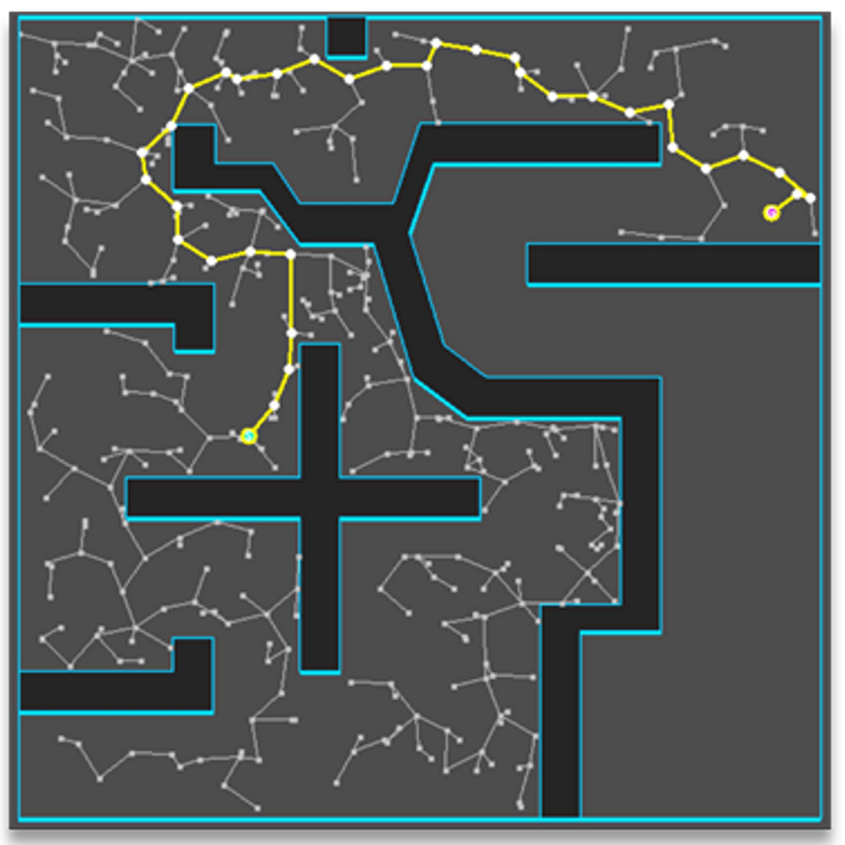

(a)

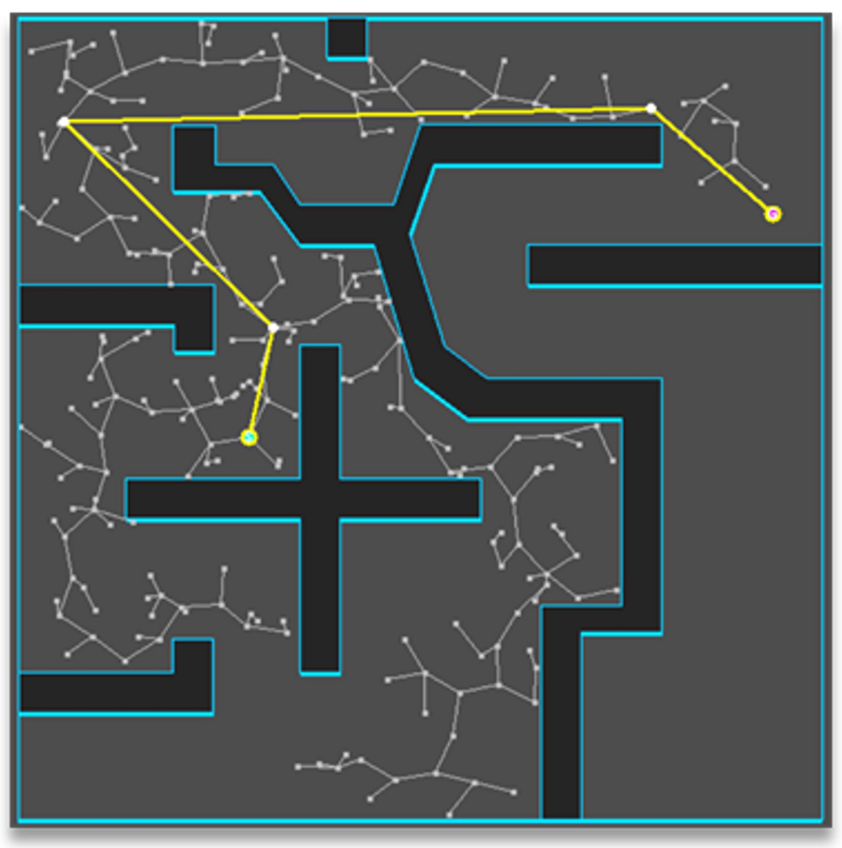

(b)

Figure 6. Experimental results of Map 2: (a) RRT and (b) proposed.

post triangular rewiring method was $82 \%$ (799/969) compared to that of the RRT, which was the shortest, and the planning time was similar.

Figure 7 depicts the path planning results for Map 3 among the presented environmental maps for each algorithm. Visually, the path of the "post triangular rewiring" method appears to be
Table 3. Experimental results of Map 2

\begin{tabular}{lcc}
\hline \multicolumn{1}{c}{ Performance } & RRT & Proposal \\
\hline Path length (pixel) & $969(100 \%)$ & $799(82 \%)$ \\
Planning time (ms) & $10(100 \%)$ & $11(100 \%)$ \\
\hline
\end{tabular}

The parentheses on the right of each number (average of repeated 100 times) are relative ratios based on $100 \%$ RRT (values less than 1 are counted as 1).

Table 4. Experimental results of Map 3

\begin{tabular}{lcc}
\hline \multicolumn{1}{c}{ Performance } & RRT & Proposal \\
\hline Path length (pixel) & $591(100 \%)$ & $529(89 \%)$ \\
Planning time (ms) & $6(100 \%)$ & $7(100 \%)$ \\
\hline
\end{tabular}

The parentheses on the right of each number (average of repeated 100 times) are relative ratios based on $100 \%$ RRT (values less than 1 are counted as 1).

Table 5. Experimental results of Map 4

\begin{tabular}{lcc}
\hline \multicolumn{1}{c}{ Performance } & RRT & Proposal \\
\hline Path length (pixel) & $1533(100 \%)$ & $1304(85 \%)$ \\
Planning time (ms) & $1526(100 \%)$ & $1527(100 \%)$ \\
\hline
\end{tabular}

The parentheses on the right of each number (average of repeated 100 times) are relative ratios based on $100 \%$ RRT (values less than 1 are counted as 1).

the shortest.

Table 4 presents the path planning results for Map 3 (average when repeated 100 times) among the presented environmental maps for each algorithm. The path length after applying the post triangular rewiring method was 89\% (529/591) compared to that of the RRT, which was the shortest, and the planning time was similar.

Figure 8 depicts the path planning results for Map 4 among the presented environmental maps for each algorithm. Visually, the path of the "post triangular rewiring" method appeared to be the shortest.

Table 5 presents the path planning results for Map 4 (average when repeated 100 times) among the presented environmental maps for each algorithm. The path length after applying the post triangular rewiring method was $85 \%$ (1304/1533) compared to that of the RRT, which was the shortest, and the planning time was similar.

Overall, the application of the "post triangular rewiring" method exhibited a good performance in the path length for all maps, indicating that the proposed method is effective in terms of optimality. 


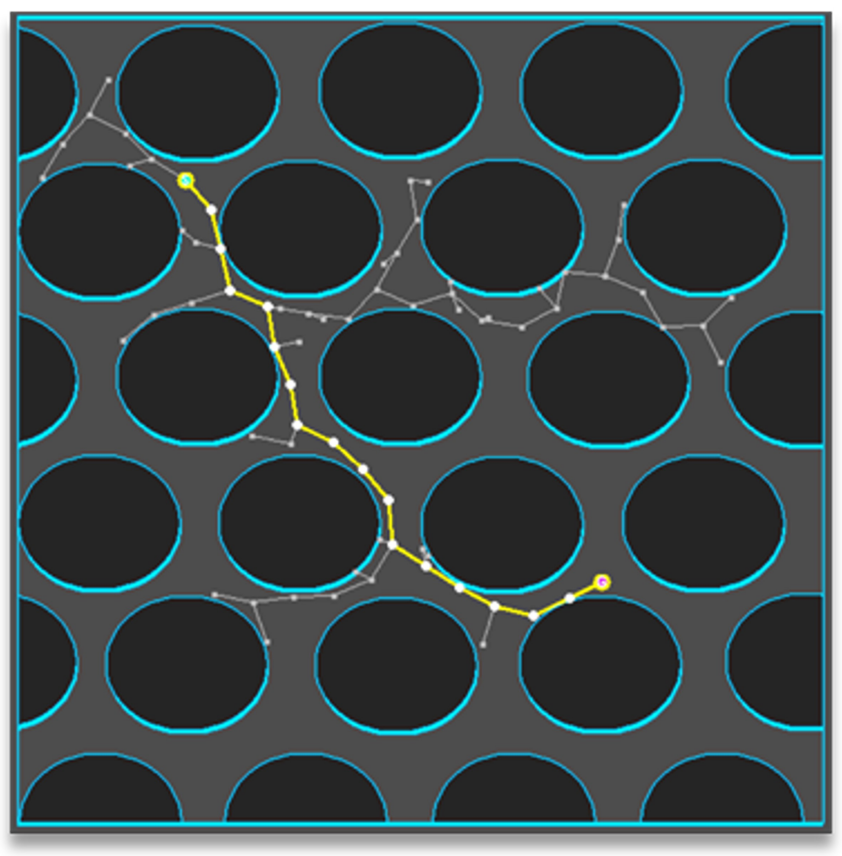

(a)

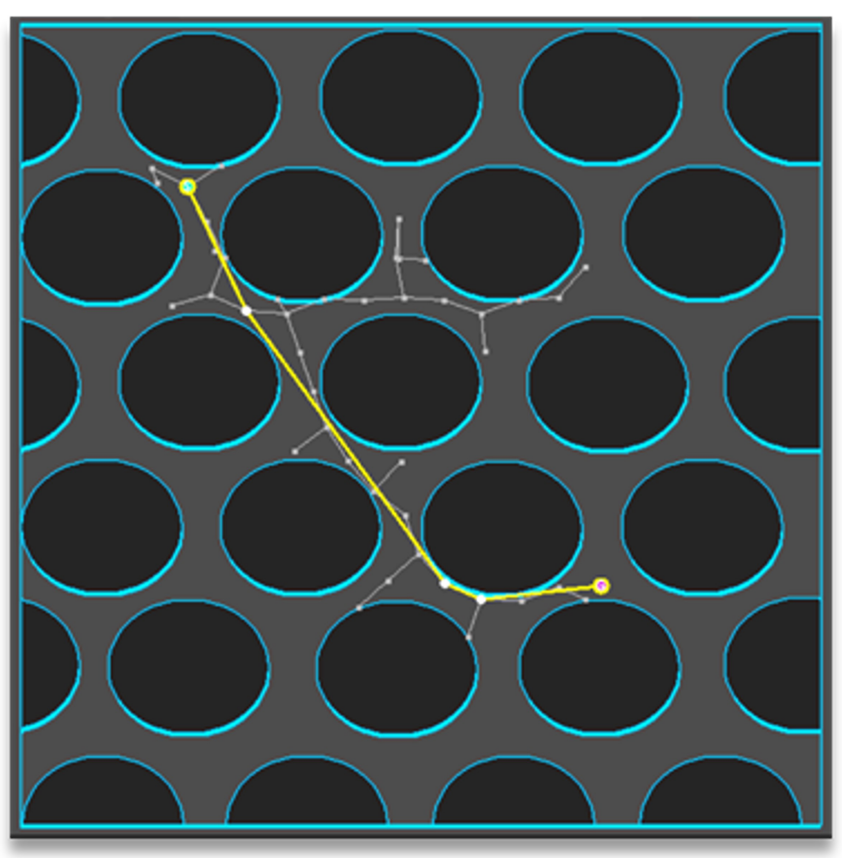

(b)

Figure 7. Experimental results of Map 3: (a) RRT and (b) proposed.

\section{Conclusion}

In this paper, we proposed a "post triangular rewiring" method that could minimize the sacrifice of planning time and overcome the limitations of the optimization of the sampling derivation

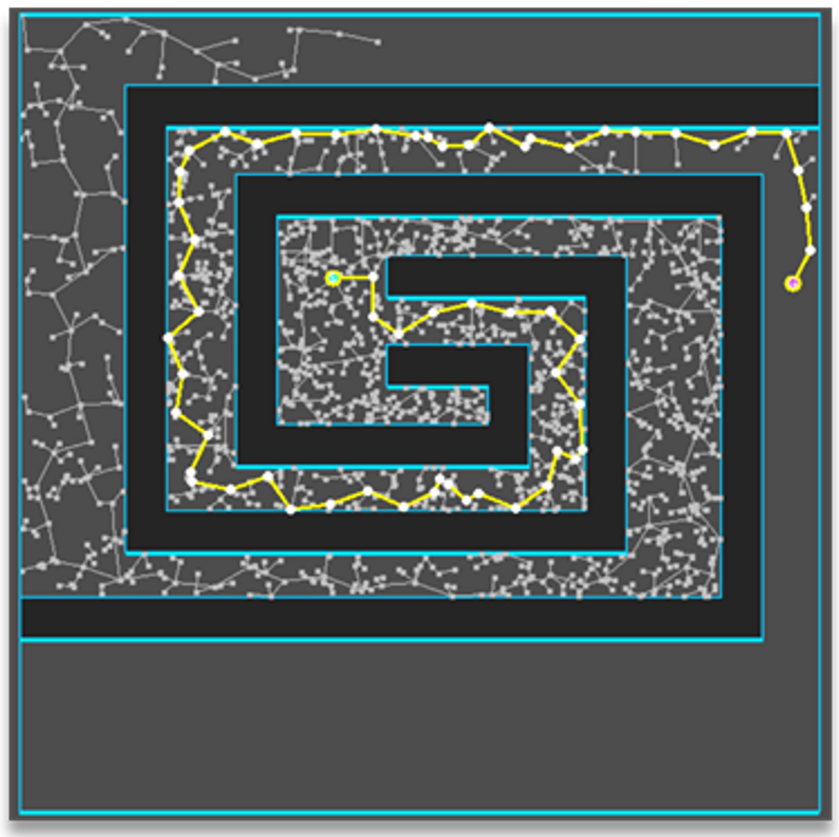

(a)

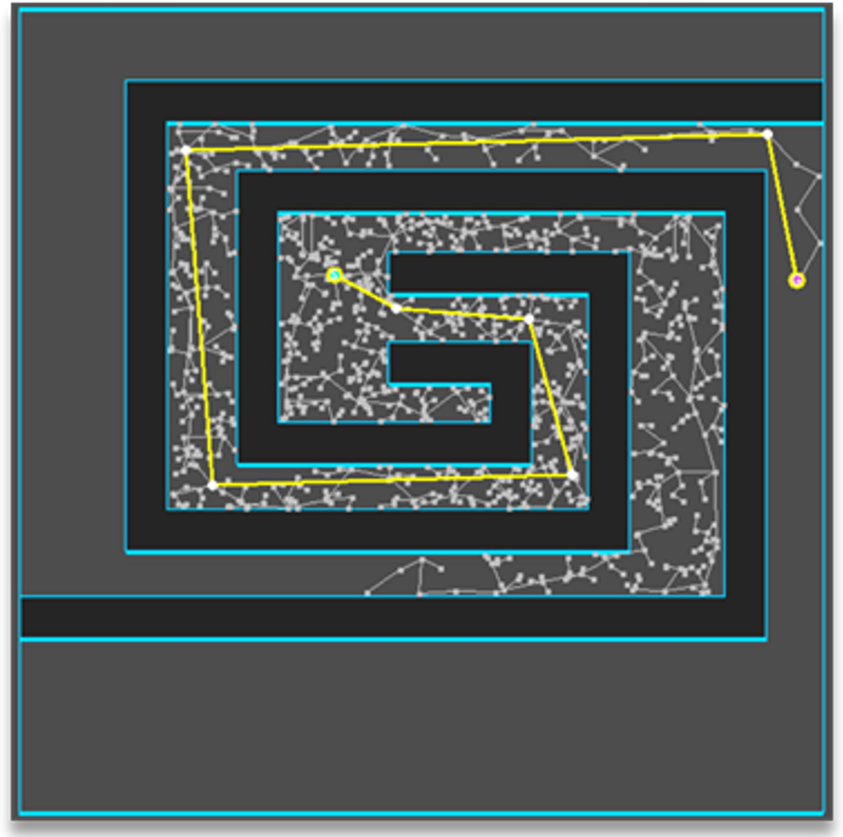

(b)

Figure 8. Experimental results of Map 4: (a) RRT and (b) proposed.

algorithm. The proposed "post triangular rewiring" method creates a more optimal path. In addition, as a post-processing method, it has the advantage of being applicable to all path planning algorithms that plan a locally piecewise linear path. Simulations were performed to verify the performance of the 
RRT algorithm, to which the post triangular rewiring method was applied. It was verified that the path length was shortened by $11-28 \%$ (average 18\%) when applied to the RRT algorithm in the four different environmental maps. Consequently, the RRT algorithm applying the proposed "post triangular rewiring" method exhibited a more optimal path.

\section{Conflict of Interest}

No potential conflict of interest relevant to this article was reported.

\section{Acknowledgement}

This work was supported by the National Research Foundation of Korea (NRF) grant funded by the Korean government (MSIT) (No. 2020R1F1A1074974), the Ministry of Trade, Industry and Energy (MOTIE), and Korea Institute for Advancement of Technology (KIAT) through the International Cooperative R\&D program (Project No. P0016096) and the Korea Association of University, Research Institute, and Industry (AURI) grant funded by the Korean Government (MSS: Ministry of SMEs and Startups) (No. S3041234, HRD program for Enterprise linkages R\&D) and the Ministry of Science and ICT (MSIT), Korea, under the Information Technology Research Center (ITRC) support program (No. IITP-2021-2020-0-01789) supervised by the Institute for Information \& Communications Technology Planning \& Evaluation (IITP).

\section{References}

[1] K. Schwab, The Fourth Industrial Revolution. Geneva, Switzerland: World Economic Forum, 2016.

[2] Y. I. Choi, J. H. Cho, and Y. T. Kim, "Collision avoidance algorithm of mobile robots at grid map intersection point," International Journal of Fuzzy Logic and Intelligent Systems, vol. 20, no. 2, pp. 96-104, 2020. https://doi.org/10.5391/IJFIS.2020.20.2.96

[3] J. H. Jung and D. H. Kim, "Local path planning of a mobile robot using a novel grid-based potential method," International Journal of Fuzzy Logic and Intelligent Systems, vol. 20, no. 1, pp. 26-34, 2020. https://doi.org/10. 5391/IJFIS.2020.20.1.26

[4] S. M. LaValle and J. J. Kuffner, "Randomized kinodynamic planning," The International Journal of Robotics
Research, vol. 20, no. 5, pp. 378-400, 2001. https://doi. org/10.1177/02783640122067453

[5] J. J. Kuffner and S. M. LaValle, "RRT-connect: an efficient approach to single-query path planning," in Proceedings of IEEE International Conference on Robotics and Automation (Cat. No. 00CH37065), San Francisco, CA, 2000, pp. 995-1001. https://doi.org/10.1109/ROBOT.2000.844730

[6] J. G. Kang, D. W. Lim, Y. S. Choi, W. J. Jang, and J. W. Jung, "Improved RRT-connect algorithm based on triangular inequality for robot path planning," Sensors, vol. 21, no. 2, article no. 333, 2021. https://doi.org/10. $3390 / \mathrm{s} 21020333$

[7] S. Karaman and E. Frazzoli, "Sampling-based algorithms for optimal motion planning," The International Journal of Robotics Research, vol. 30, no. 7, pp. 846-894, 2011. https://doi.org/10.1177/0278364911406761

[8] J. D. Gammell, S. S. Srinivasa, and T. D. Barfoot, "Informed RRT*: optimal sampling-based path planning focused via direct sampling of an admissible ellipsoidal heuristic," in Proceedings of 2014 IEEE/RSJ International Conference on Intelligent Robots and Systems, Chicago, IL, 2004, pp. 2997-3004. https://doi.org/10.1109/IROS. 2014.6942976

[9] S. Klemm, J. Oberlander, A. Hermann, A. Roennau, T. Schamm, J. M. Zollner, and R. Dillmann, "RRT*Connect: faster, asymptotically optimal motion planning," in Proceedings of 2015 IEEE international Conference on Robotics and Biomimetics (ROBIO), Zhuhai, China, 2015, pp. 1670-1677. https://doi.org/10.1109/ROBIO. 2015.7419012

[10] F. Islam, J. Nasir, U. Malik, Y. Ayaz, and O. Hasan, "RRT*-Smart: rapid convergence implementation of RRT* towards optimal solution," in Proceedings of 2012 IEEE International Conference on Mechatronics and Automation, Chengdu, China, 2012, pp. 1651-1656. https://doi. org/10.1109/ICMA.2012.6284384

[11] D. Roy, "Visibility graph-based spatial path planning of robots using configuration space algorithms," International Journal of Robotics \& Automation, vol. 24, no. 1, article no. 2853, 2019. http://doi.org/10.2316/Journal. 206.2009.1.206-2853 
[12] N. I. Katevas, S. G. Tzafestas, and C. G. Pnevmatikatos, "The approximate cell decomposition with local node refinement global path planning method: path nodes refinement and curve parametric interpolation," Journal of Intelligent and Robotic Systems, vol. 22, no. 3, pp. 289-314, 1998. https://doi.org/10.1023/A:1008034314006

[13] C. W. Warren, "Global path planning using artificial potential fields," in Proceedings of 1989 IEEE International Conference on Robotics and Automation, Scottsdale, AZ, 1989, pp. 316-317. https://doi.org/10.1109/ROBOT.1989. 100007

[14] S. M. LaValle, "Rapidly-exploring random trees: A new tool for path planning," Department of Computer Science, Iowa State University, Ames, IA, 1998.

[15] J. Han and Y. Seo, "Mobile robot path planning with surrounding point set and path improvement," Applied Soft Computing, vol. 57, pp. 35-47, 2017. https://doi.org/ 10.1016/j.asoc.2017.03.035

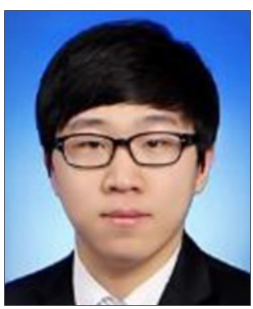

Jin-Gu Kang received the B.S. and LL.B. degrees from Dongguk University at Seoul, Korea in 2019, and received the M.S. degree in Computer Science and Engineering from Dongguk University at Seoul, Korea in 2021. His research areas include robot path planning, wireless MAC protocols and deep learning-based image processing.

E-mail: kanggu12@dongguk.edu

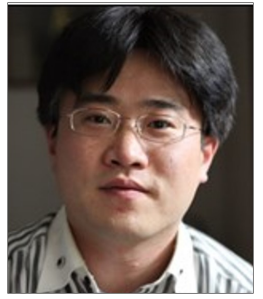

Jin-Woo Jung received the B.S. and M.S. degrees in Electrical Engineering from Korea Advanced Institute of Science and Technology (KAIST), Korea, in 1997 and 1999, respectively and received the Ph.D. degree in Electrical Engineering and Computer Science from KAIST, Korea in 2004. Since 2006, he has been with the Department of Computer Science and Engineering at Dongguk University at Seoul, Korea, where he is currently a professor. His current research interests include human behavior recognition, mobile robot and intelligent human-robot interaction

E-mail: jwjung@dongguk.edu 